\title{
Periodic Orbits with Least Period Three on the Circle
}

\author{
Xuezhi Zhao \\ Department of Mathematics, Capital Normal University, Beijing 100037, China \\ Correspondence should be addressed to Xuezhi Zhao, zhaoxve@mail.cnu.edu.cn \\ Received 19 August 2007; Accepted 3 December 2007 \\ Recommended by J. R. L. Webb \\ The self-maps on the circle having periodic orbits with least period 3 are classified into relative \\ homotopy-conjugacy classes. We will show that except for the maps in one exceptional class, all \\ these kinds of maps have periodic orbits with all least periods but 2. Thus, a kind of Sharkovskii's \\ type theorem on the circle is obtained.
}

Copyright (c) 2008 Xuezhi Zhao. This is an open access article distributed under the Creative Commons Attribution License, which permits unrestricted use, distribution, and reproduction in any medium, provided the original work is properly cited.

\section{Introduction}

Because of [1], it is well known that a map on the interval must have periodic points of each least period if it has a periodic point of least period 3. This result was actually a special case of Sharkovskii's theorem [2]. Its various generalizations became a rich and active field in mathematics (see [3]).

It is very natural to ask for an analogy of Sharkovskii's theorem for maps on the circle. Unfortunately, the answer is negative if the period of a periodic orbit is given merely. For any positive integer $n$, the $360 / n$ degree rotation admits all points as periodic points with least period $n$, but has no periodic point with any other least periods. Thus, one needs more information about the given periodic orbits in order to obtain other periodic points. In [4], the existence of periodic points was obtained according to the homotopy classes, that is, the degrees, of maps on the circle. More general, combinatorial structures of given period orbits of maps on graphs (as opposed to merely their periods) were formulated as the "pattern" $[5,6]$.

In this note, by combining algebraic topology and dynamical system approaches, we will classify relative maps (i.e., maps with invariant subsets) into some relative homotopyconjugacy classes. Such an idea is an extension of those in $[5,7]$. Especially, consider the maps 
on the circle having periodic orbits with least period 3, we will show that except for the maps in one relative homotopy-conjugacy class, all maps have periodic points with least period $n$ for all positive integer $n \neq 2$, where the given periodic orbits are regarded as their finite invariant subsets. Our main tool is the relative Nielsen fixed point theory. At the end, we present some examples showing that our conclusion is not true if the least period of the given periodic orbit is larger than 5 .

This paper is organized as follows. In Section 2, we will review some results in relative Nielsen fixed point theory, which will be used here. A new classification of relative maps, namely, "relative homotopy-conjugacy classes" will be defined in Section 3. Our main result will be given in Section 4. In Section 5, we illustrate some examples when the given period is larger than 5 .

\section{Surplus fixed point classes}

In this section, we will review some definitions and results in relative Nielsen fixed point theory, especially those related to our purpose here; see [8] for more details and [9] for general relative Nielsen fixed point theory.

Consider a map $f: X \rightarrow X$ on a compact connected polyhedron $X$. Let $p: \tilde{X} \rightarrow X$ be the universal covering of $X$. A map $\tilde{f}: \tilde{X} \rightarrow \tilde{X}$ is said to be a lifting of $f$ if the following diagram commutes:

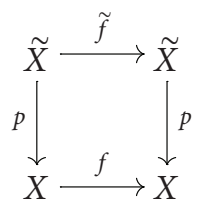

Let $U$ be a path-connected subset of $X$. Fix a component $\tilde{U}$ of $p^{-1}(U)$, we have

$$
U \cap \operatorname{Fix}(f)=\bigcup_{\tilde{f}} p(\tilde{U} \cap \operatorname{Fix}(\tilde{f}))
$$

where $\tilde{f}$ ranges over all liftings of $f$. According to [8, Definition 2.1], two pairs $(\tilde{f}, \tilde{U})$ and $\left(\tilde{f}^{\prime}, \tilde{U}^{\prime}\right)$ of $f$ on $U$ are said to be conjugate if there exists a covering translation $\gamma$ such that $\gamma(\tilde{U})=\tilde{U}^{\prime}$ and $\tilde{f}^{\prime}=\gamma \circ \tilde{f} \circ \gamma^{-1}$.

Proposition 2.1. For any two pairs $(\tilde{f}, \tilde{U})$ and $\left(\tilde{f}^{\prime}, \tilde{U}^{\prime}\right)$ of $f$ on $U$, either $p(\tilde{U} \cap \operatorname{Fix}(\tilde{f})) \cap p\left(\tilde{U}^{\prime} \cap\right.$ $\left.\operatorname{Fix}\left(\tilde{f}^{\prime}\right)\right)=\varnothing$, if $(\tilde{f}, \tilde{U})$ and $\left(\tilde{f}^{\prime}, \tilde{U}^{\prime}\right)$ are not conjugate, or $p(\tilde{U} \cap \operatorname{Fix}(\tilde{f}))=p\left(\tilde{U}^{\prime} \cap \operatorname{Fix}\left(\tilde{f}^{\prime}\right)\right)$, if $(\tilde{f}, \tilde{U})$ and $\left(\tilde{f}^{\prime}, \tilde{U}^{\prime}\right)$ are conjugate.

The subset $p(\tilde{U} \cap \operatorname{Fix}(\tilde{f}))$ of the fixed point set of $f$ on $U$ is said to be the fixed point class of $f$ on $U$ being determined by the pair $(\tilde{f}, \tilde{U})$. By the above proposition, the fixed point set $U \cap \operatorname{Fix}(f)$ of $f$ on $U$ splits into a disjoint union of the fixed point classes of $f$ on $U$. Let $f:(X, A) \rightarrow(X, A)$ be a relative map on a pair of compact polyhedra. One can consider the fixed point classes of $f$ on the components of $X-A$ in the sense above. Each of these fixed point classes is said to be a fixed point class of $f$ on $X-A$. 
Definition 2.2 (see [10, Definition 3.1] and [8, Definition 4.7]). Let $F$ be a fixed point class of $f$ on $X-A$ which is determined by $(\tilde{f}, \tilde{C})$, where $\widetilde{C}$ is a component of $p^{-1}(X-A)$. The fixed point class $F$ is said to be nonsurplus if there is a component $\tilde{A}$ of $p^{-1}(A)$ with $\tilde{A} \cap \mathrm{cl}(\tilde{C}) \neq \varnothing$ such that $\tilde{f}(\tilde{A}) \subset \widetilde{A}$ is said to be surplus if it is not nonsurplus.

Of most importance is that any surplus fixed point class is a compact set, and hence has a well-defined index. The so-called surplus Nielsen number $\mathrm{SN}(f ; X-A)$ of a relative map $f:(X, A) \rightarrow(X, A)$ on $X-A$ is defined as the number of essential surplus fixed point classes of $f$ on $X-A$.

\section{Relative homotopy-conjugacy classes}

Here, we define a new classification of relative self maps, which is just a combination of relative homotopy classes and relative topological conjugacy classes.

Definition 3.1. Two relative maps $f:(X, A) \rightarrow(X, A)$ and $f^{\prime}:\left(X, A^{\prime}\right) \rightarrow\left(X, A^{\prime}\right)$ are said to be relatively homotopy-conjugate if there is a finite sequence of relative maps: $f_{k}:\left(X, A_{k}\right)$ $\rightarrow\left(X, A_{k}\right), k=0,1,2, \ldots, n$, with $A_{0}=A, A_{n}=A^{\prime}, f_{0}=f$, and $f_{n}=f^{\prime}$ such that for each $k$, $k=1,2, \ldots, n$.

Either (1) $f_{k-1}$ and $f_{k}$ are relatively homotopic, that is, $A_{k-1}=A_{k}$ and there is a relative homotopy $H:\left(X \times I, A_{k} \times I\right) \rightarrow\left(X, A_{k}\right)$ such that $H(x, 0)=f_{k-1}(x)$ and $H(x, 1)=f_{k}(x)$ for all $x \in X$.

Or (2) $f_{k-1}$ and $f_{k}$ are relatively conjugate, that is, there is a relative homeomorphism $h_{k}$ such that the following diagram commutes:

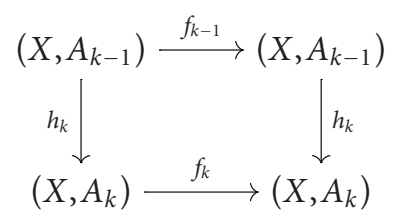

Clearly, "relative homotopy-conjugacy" is an equivalent relation in the set of relative maps on homeomorphic space pairs. Each equivalent class is said to be a relative homo topy conjugacy class. In the case of circle maps, a similar relation, a little restricted, was named as "h-equivalent" (see [5, page179]).

Lemma 3.2. If two relative maps $f:(X, A) \rightarrow(X, A)$ and $f^{\prime}:\left(X, A^{\prime}\right) \rightarrow\left(X, A^{\prime}\right)$ are relatively homotopy-conjugate. Then $\mathrm{SN}(f ; X-A)=\mathrm{SN}\left(f^{\prime} ; X-A^{\prime}\right)$.

Proof. It is sufficient to show that the surplus Nielsen number is invariant under relative homotopy and relative conjugacy. The first part was proved in [10, Theorem 3.6], and the second part was given in [11, Proposition 3.11].

Since any essential surplus fixed point class is always nonempty, we obtain the following theorem.

Theorem 3.3. Any relative map $f^{\prime}:\left(X, A^{\prime}\right) \rightarrow\left(X, A^{\prime}\right)$ which is relatively homotopyconjugate to a relative map $f:(X, A) \rightarrow(X, A)$ has at least $\mathrm{SN}(f ; X-A)$ fixed points on $X-A^{\prime}$. 
This lower-bound property enables us to prove simultaneously the existence of fixed points for all maps in a given relative homotopy-conjugacy class, instead of proving their existence individually.

\section{Circle maps with periodic points of least period 3}

In this section, we will consider the maps on the circle which have periodic points with least period 3. Let us fix some notations.

For a triple $\left(n_{0}, n_{1}, n_{2}\right)$ of integers, we define a relative map $f_{\left(n_{0}, n_{1}, n_{2}\right)}: S^{1} \rightarrow S^{1}$ by

$$
f_{\left(n_{0}, n_{1}, n_{2}\right)}\left(e^{\theta i}\right)=\exp \left(2 \pi \lambda_{\left(n_{0}, n_{1}, n_{2}\right)}\left(\frac{\theta}{2 \pi}\right) i\right), \quad 0 \leq \theta<2 \pi
$$

where the map $\lambda_{\left(n_{0}, n_{1}, n_{2}\right)}:[0,1) \rightarrow R^{1}$ is defined by

$$
\lambda_{\left(n_{0}, n_{1}, n_{2}\right)}(x)= \begin{cases}6 x+\frac{1}{3} & \text { if } 0 \leq x \leq \frac{1}{9}, \\ 9 n_{0} x-n_{0}+1 & \text { if } \frac{1}{9} \leq x \leq \frac{2}{9} \\ -3 x+n_{0}+1+\frac{2}{3} & \text { if } \frac{2}{9} \leq x \leq \frac{1}{3} \\ 3 x+n_{0}-\frac{1}{3} & \text { if } \frac{1}{3} \leq x \leq \frac{4}{9} \\ 9 n_{1} x+n_{0}+1-4 n_{1} & \text { if } \frac{4}{9} \leq x \leq \frac{5}{9} \\ n_{0}+n_{1}+1 & \text { if } \frac{5}{9} \leq x \leq \frac{7}{9} \\ 9 n_{2} x+n_{0}+n_{1}+1-7 n_{2} & \text { if } \frac{7}{9} \leq x \leq \frac{8}{9} \\ 3 x+n_{0}+n_{1}+n_{2}-\frac{5}{3} & \text { if } \frac{8}{9} \leq x<1 .\end{cases}
$$

We may regard $S^{1}$ as a graph with three vertices $v_{0}, v_{1}, v_{2}$ and three edges $w_{0}, w_{1}, w_{2}$, where $w_{k}=\left\{e^{\theta i} \mid 2 k \pi / 3 \leq \theta \leq(2 k+2) \pi / 3\right\}$ and $v_{k}=e^{(2 k \pi / 3) i}, k=0,1,2$. Thus, $f_{\left(n_{0}, n_{1}, n_{2}\right)}$ is a relative self-map on the pair $\left(S^{1}, V=\left\{v_{0}, v_{1}, v_{2}\right\}\right)$ satisfying

$$
\begin{aligned}
& w_{0} \longmapsto w_{1} w_{2}\left(w_{0} w_{1} w_{2}\right)^{n_{0}} w_{2}^{-1}, \\
& w_{1} \longmapsto w_{2}\left(w_{0} w_{1} w_{2}\right)^{n_{1}}, \\
& w_{2} \longmapsto\left(w_{0} w_{1} w_{2}\right)^{n_{2}} w_{0} .
\end{aligned}
$$

Consider the universal covering $p: R^{1} \rightarrow S^{1}$, which is defined by $p(x)=e^{2 \pi x i}$. The set of all liftings of $f_{\left(n_{0}, n_{1}, n_{2}\right)}$ is

$$
\left\{\tilde{f}_{\left(n_{0}, n_{1}, n_{2}\right), m} \mid m=0, \pm 1, \pm 2, \ldots\right\},
$$

where $\tilde{f}_{\left(n_{0}, n_{1}, n_{2}\right), m}: R^{1} \rightarrow R^{1}$ is defined by

$$
\tilde{f}_{\left(n_{0}, n_{1}, n_{2}\right), m}(x)=\lambda_{\left(n_{0}, n_{1}, n_{2}\right)}(x-[x])+\left(n_{0}+n_{1}+n_{2}+1\right)[x]+m,
$$

in which $[x]$ is the maximal integer less or equal to $x$. 
Lemma 4.1. Let $f_{\left(n_{0}, n_{1}, n_{2}\right)}:\left(S^{1}, V\right) \rightarrow\left(S^{1}, V\right)$ be the map as above. Then $\mathrm{SN}\left(f_{\left(n_{0}, n_{1}, n_{2}\right)} ; S^{1}-V\right)=$ $\left|n_{0}\right|+\left|n_{1}\right|+\left|n_{2}\right|$.

Proof. Since $f_{\left(n_{0}, n_{1}, n_{2}\right)}:\left(S^{1}, V\right) \rightarrow\left(S^{1}, V\right)$ does not send any component (point) of $V$ into itself, $\tilde{f}_{\left(n_{0}, n_{1}, n_{2}\right), m}(x) \neq x$ for any lifting $\tilde{f}_{\left(n_{0}, n_{1}, n_{2}\right), m}$ (see (4.5) for definition) of $f_{\left(n_{0}, n_{1}, n_{2}\right)}$ and any $x \in$ $p^{-1}(V)$. By definition, any fixed point class of $f$ on $S^{1}-V$ is surplus.

Note that $S^{1}-V$ has three components: $\dot{w}_{0}, \dot{w}_{1}$, and $\dot{w}_{2}$, which are, respectively, the interiors of $w_{0}, w_{1}$, and $w_{2}$. By a computation, any fixed point of $f_{\left(n_{0}, n_{1}, n_{2}\right)}$ on $\dot{w}_{0}$ has the form $\exp \left(\left(\left(2 n_{0}-2-2 m\right) /\left(9 n_{0}-1\right)\right) \pi i\right)$ for some integer $m$. Thus,

$$
\operatorname{Fix}\left(f_{\left(n_{0}, n_{1}, n_{2}\right)}\right) \cap \dot{w}_{0}= \begin{cases}\left\{\exp \left(\frac{2 n_{0}-2-2 m}{9 n_{0}-1} \pi i\right) \mid m=-1,-2, \ldots,-n_{0}\right\} & \text { if } n_{0}>0 \\ \varnothing & \text { if } n_{0}=0 \\ \left\{\exp \left(\frac{2 n_{0}-2-2 m}{9 n_{0}-1} \pi i\right) \mid m=0,1,2, \ldots,-n_{0}-1\right\} & \text { if } n_{0}<0 .\end{cases}
$$

Note that the fixed point $\exp \left(\left(\left(2 n_{0}-2-2 m\right) /\left(9 n_{0}-1\right)\right) \pi i\right)$ is the projection of the fixed point of $\tilde{f}_{\left(n_{0}, n_{1}, n_{2}\right), m}$ on the component $(0,2 / 3)$ of $p^{-1}\left(\dot{w}_{0}\right)$, that is,

$$
\exp \left(\frac{2 n_{0}-2-2 m}{9 n_{0}-1} \pi i\right)=p\left(\left(0, \frac{2}{3}\right) \cap \operatorname{Fix}\left(\tilde{f}_{\left(n_{0}, n_{1}, n_{2}\right), m}\right)\right)
$$

By definition of fixed point class (see Proposition 2.1), those $\left|n_{0}\right|$ fixed points lie in different fixed point classes of $f_{\left(n_{0}, n_{1}, n_{2}\right)}$ on $S^{1}-V$. It is obvious that each of these $\left|n_{0}\right|$ fixed point classes has index $\operatorname{sgn}\left(\operatorname{det}\left(1-D f_{*}\right)\right)=\operatorname{sgn}\left(1-9 n_{0}\right)=-\operatorname{sgn}\left(n_{0}\right)$, and hence is essential.

Similarly, $f_{\left(n_{0}, n_{1}, n_{2}\right)}$ has $\left|n_{1}\right|$ essential fixed point classes on $\dot{w}_{1}$, and has $\left|n_{2}\right|$ essential fixed point classes on $\dot{w}_{2}$. So, we are done.

Next two lemmas will show that these $f_{\left(n_{0}, n_{1}, n_{2}\right)}$ 's can be regarded as representations of maps on the circle having periodic points with least period 3.

Lemma 4.2. Two maps $f_{\left(n_{0}, n_{1}, n_{2}\right)}$ and $f_{\left(n_{0}^{\prime}, n_{1}^{\prime}, n_{2}^{\prime}\right)}$ are in the same relative homotopy-conjugacy class if and only if $\left(n_{0}, n_{1}, n_{2}\right)$ and $\left(n_{0}^{\prime}, n_{1}^{\prime}, n_{2}^{\prime}\right)$ are the same up to a permutation.

Proof. It is a straight verification.

Lemma 4.3. Let $f$ be a self-map on $S^{1}$. If $f$ has a periodic orbit $P$ with least period 3 , then as a relative map, $f:\left(S^{1}, P\right) \rightarrow\left(S^{1}, P\right)$ is relatively homotopy-conjugate to a map $f_{\left(n_{0}, n_{1}, n_{2}\right)}:\left(S^{1}, V\right) \rightarrow\left(S^{1}, V\right)$ for some integers $n_{0}, n_{1}, n_{2}$.

Proof. Let $P=\left\{x_{0}, x_{1}, x_{2}\right\}$. Since $P$ is a periodic orbit with least period 3 , we may assume that $x_{1}=f\left(x_{0}\right), x_{2}=f^{2}\left(x_{0}\right)$, and $x_{0}=f^{3}\left(x_{0}\right)$. Note that the three points in $P$ are distinct. There is a homeomorphism $h: S^{1} \rightarrow S^{1}$ such that $h\left(x_{k}\right)=v_{k}, k=0,1,2$. The relative map $h \circ f \circ h^{-1}:\left(S^{1}, V\right) \rightarrow\left(S^{1}, V\right)$ is therefore relatively conjugate to $f:\left(S^{1}, P\right) \rightarrow\left(S^{1}, P\right)$.

Consider the universal covering $p: R^{1} \rightarrow S^{1}$. By using the notation at the beginning of this section, we have $p(0)=v_{0}$ and $p(1 / 3)=v_{1}$. Since $h \circ f \circ h^{-1}\left(v_{0}\right)=v_{1}$, there is a unique lifting $\tilde{f}^{\prime}$ of $h \circ f \circ h^{-1}$ such that $\tilde{f}^{\prime}(0)=1 / 3$. As $h \circ f \circ h^{-1}\left(v_{1}\right)=v_{2}$, it follows that $\tilde{f}^{\prime}(1 / 3) \in p^{-1}\left(v_{2}\right)$, and 
hence $\tilde{f}^{\prime}(1 / 3)=2 / 3+n_{0}$ for some integer $n_{0}$. Note that $R^{1}$ is simply connected. $\left.\tilde{f}^{\prime}\right|_{[0,1 / 3]}$ and $\left.\lambda_{\left(n_{0}, n_{1}, n_{2}\right)}\right|_{[0,1 / 3]}$ are homotopic relative to $\{0,1 / 3\}$ and form any integers $n_{1}$ and $n_{2}$. Project this homotopy down to $S^{1}$, we will have a homotopy, keeping $v_{0}$ and $v_{1}$ fixed, from $h \circ f \circ h^{-1} \mid w_{0}$ to $f_{\left(n_{0}, n_{1}, n_{2}\right)} \mid w_{0}$ for any integers $n_{1}$ and $n_{2}$. Repeat this argument at $\left.h \circ f \circ h^{-1}\right|_{w_{1}}$ and $\left.h \circ f \circ h^{-1}\right|_{w_{2}}$, we will obtain an integer $n_{1}$ satisfying $\tilde{f}^{\prime}(2 / 3)=n_{0}+n_{1}+1$, and an integer $n_{2}$ satisfying $\tilde{f}^{\prime}(1)=n_{0}+n_{1}+n_{2}+4 / 3$. Thus, $\left.\tilde{f}^{\prime}\right|_{[0,1]}$ is homotopic, relative $\{0,1 / 3,2 / 3,1\}$, to $\left.\tilde{f}_{\left(n_{0}, n_{1}, n_{2}\right), 0}\right|_{[0,1]}$. Project down to $S^{1}$, it follows that $h \circ f \circ h^{-1}$ is relatively homotopic to $f_{\left(n_{0}, n_{1}, n_{2}\right)}$, and therefore $f:\left(S^{1}, P\right) \rightarrow\left(S^{1}, P\right)$ is relatively homotopy-conjugate to $f_{\left(n_{0}, n_{1}, n_{2}\right)}:\left(S^{1}, V\right) \rightarrow\left(S^{1}, V\right)$.

We restate a result of L. Block as follows.

Lemma 4.4 (see [12, Theorem A]). Let $f$ be a self map on $S^{1}$. Suppose that $f$ has a fixed point and a periodic point with least period $n(n>1)$. Then one of the following holds:

(i) $f$ has a periodic point with least period $m$ for every $m>n$,

(ii) $f$ has a periodic point with least period $m$ for every $m$ satisfying $n>_{s} m$, where $>_{s}$ is Sharkovskii's order of natural number set given by

$$
3>_{s} 5>_{s} 7>_{s} \cdots>_{s} 2 \cdot 3>_{s} 2 \cdot 5>_{s} 2 \cdot 7>_{s} \cdots>_{s} 8>_{s} 4>_{s} 2>_{s} 1 \text {. }
$$

Our main result is the following theorem.

Theorem 4.5. Let $f$ be a self map on $S^{1}$ having a periodic orbit $P$ with least period 3. Then $f$ has periodic points of each least period except for 2 if it is not relatively homotopy-conjugate to $f_{(0,0,0)}$ : $\left(S^{1}, V\right) \rightarrow\left(S^{1}, V\right)$, which is the standard 120 degree rotation.

Proof. By Lemma 4.3, $f:\left(S^{1}, P\right) \rightarrow\left(S^{1}, P\right)$ is relatively homotopy-conjugate to a relative map $f_{\left(n_{0}, n_{1}, n_{2}\right)}:\left(S^{1}, V\right) \rightarrow\left(S^{1}, V\right)$. From Lemmas 3.2 and 4.1, we have that $S N\left(f ; S^{1}-P\right)=\left|n_{0}\right|+\left|n_{1}\right|+$ $\left|n_{2}\right|$. Since $\left(n_{0}, n_{1}, n_{2}\right) \neq(0,0,0), \mathrm{SN}\left(f ; S^{1}-P\right)>0$. It follows that $f$ has a fixed point on $S^{1}-P$. By using Lemma 4.4 in the case $n=3, f$ has periodic points with each least period except for least period 2.

According to relative homotopy-conjugacy classes, period 3 on the circle almost forces all the other periods with only one exception. Roughly speaking, the statement that period 3 implies every period is almost true for maps on the circle. In some sense, our results cannot be improved because the map $f_{(-1,-1,-1)}$ has no periodic points with least period 2 .

Our statements here give more information about the coexistence of periodic points on the circle, comparing with the results in $[4,5,12]$, and so on. The relative homotopy-conjugacy classes refine the homotopy classes, indicated by degree, on maps on the circle, because of the following.

Proposition 4.6. The degree of $f_{\left(n_{0}, n_{1}, n_{2}\right)}$ is $n_{0}+n_{1}+n_{2}+1$.

In fact, our improvement lies in the cases that the degree of a given map is $-1,0$, or 1 . It was already known from [4] that a map on the circle has a periodic point of any least period if its degree is not $-2,-1,0$, or 1 and has a periodic point of any least period except for 2 if its degree is -2 . 


\section{Periodic orbits with larger periods}

Finally, we illustrate some examples to show what will happen if the least period of a given periodic orbit is larger than 5 .

Example 5.1. Let $m$ be an integer with $m \geq 5$, map $g_{m}^{\prime}: S^{1} \rightarrow S^{1}$ is defined by

$$
g_{m}^{\prime}\left(e^{\theta i}\right)= \begin{cases}\exp \left(\left(3 \theta+\frac{4 \pi}{m}\right) i\right) & \text { if } 0 \leq \theta \leq \frac{2 \pi}{m}, \\ \exp \left(\left(-2 \theta+\frac{14 \pi}{m}\right) i\right) & \text { if } \frac{2 \pi}{m} \leq \theta \leq \frac{4 \pi}{m}, \\ \exp \left(\left(\theta+\frac{2 \pi}{m}\right) i\right) & \text { if } \frac{4 \pi}{m} \leq \theta \leq \frac{6 \pi}{m}, \\ \exp \left(\left(2 \theta-\frac{4 \pi}{m}\right) i\right) & \text { if } \frac{6 \pi}{m} \leq \theta \leq \frac{8 \pi}{m}, \\ \exp \left(\left(\theta+\frac{4 \pi}{m}\right) i\right) & \text { if } \frac{8 \pi}{m} \leq \theta<2 \pi,\end{cases}
$$

and $\operatorname{map} g_{m}^{\prime \prime}: S^{1} \rightarrow S^{1}$ is defined by

$$
g_{m}^{\prime \prime}\left(e^{\theta i}\right)= \begin{cases}\exp \left(\left(2 \theta+\frac{4 \pi}{m}\right) i\right) & \text { if } 0 \leq \theta \leq \frac{2 \pi}{m} \\ \exp \left(\left(-\theta+\frac{10 \pi}{m}\right) i\right) & \text { if } \frac{2 \pi}{m} \leq \theta \leq \frac{4 \pi}{m} \\ \exp \left(\left(2 \theta-\frac{2 \pi}{m}\right) i\right) & \text { if } \frac{4 \pi}{m} \leq \theta \leq \frac{6 \pi}{m} \\ \exp \left(\left(\theta+\frac{4 \pi}{m}\right) i\right) & \text { if } \frac{6 \pi}{m} \leq \theta<2 \pi .\end{cases}
$$

If $m$ is odd and larger than $5, g_{m}^{\prime}$ has a periodic orbit with least period $m$ :

$$
\begin{gathered}
V^{\prime}=\left\{e^{0 i}, e^{(4 \pi / m) i}, e^{(6 \pi / m) i}, e^{(8 \pi / m) i}, e^{(12 \pi / m) i}, \ldots, e^{(2(m-1) \pi / m) i},\right. \\
\left.e^{(2 \pi / m) i}, e^{(10 \pi / m) i}, e^{(14 \pi / m) i}, \ldots, e^{(2(m-2) \pi / m) i}\right\} .
\end{gathered}
$$

It is evident that $g_{m}^{\prime}:\left(S^{1}, V^{\prime}\right) \rightarrow\left(S^{1}, V^{\prime}\right)$ is not relatively homotopy-conjugate to any standard rotation, but has no fixed point.

If $m$ is even and larger than $4, g_{m}^{\prime \prime}$ has a periodic orbit with least period $m$ :

$$
\begin{gathered}
V^{\prime \prime}=\left\{e^{0 i}, e^{(4 \pi / m) i}, e^{(6 \pi / m) i}, e^{(10 \pi / m) i}, e^{(14 \pi / m) i}, \ldots, e^{(2(m-1) \pi / m) i},\right. \\
\left.e^{(2 \pi / m) i}, e^{(8 \pi / m) i}, e^{(12 \pi / m) i}, \ldots, e^{(2(m-2) \pi / m) i}\right\} .
\end{gathered}
$$

It is evident that $g_{m}^{\prime \prime}:\left(S^{1}, V^{\prime \prime}\right) \rightarrow\left(S^{1}, V^{\prime \prime}\right)$ is not relatively homotopy-conjugate to any standard rotation, but has no fixed point.

This example implies that except for nonrotation condition, more hypotheses are necessary to force a fixed point on the circle when we are given a periodic orbit with a larger least period.

Recall from the proof of Theorem 4.5 that the existence of a fixed point is the key point to have periodic points of other least periods, that is, to apply Lemma 4.4. Thus, Example 5.1 shows that the proof of Theorem 4.5 does not work if the least period of given periodic orbit is larger than 5 . 


\section{Acknowledgment}

This work is supported by NSF of China (10771143) and a BMEC grant (KZ 200710025012).

\section{References}

[1] T. Y. Li and J. A. Yorke, "Period three implies chaos," The American Mathematical Monthly, vol. 82, no. 10, pp. 985-992, 1975.

[2] O. M. Sarkovskii, "Co-existence of cycles of a continuous mapping of the line into itself," Ukrainski Matematicheskiน Zhurnal, vol. 16, no. 1, pp. 61-71, 1964 (Russian).

[3] M. Misiurewicz, "Thirty years after Sharkovskiı̌'s theorem," International Journal of Bifurcation and Chaos in Applied Sciences and Engineering, vol. 5, no. 5, pp. 1275-1281, 1995.

[4] L. Block, J. Guckenheimer, M. Misiurewicz, and L. S. Young, "Periodic points and topological entropy of one-dimensional maps," in Global Theory of Dynamical Systems. Proceedings of the International Conference held at Northwestern University, Evanston, Ill., 1979, vol. 819 of Lecture Notes in Mathematics, pp. 18-34, Springer, Berlin, Germany, 1980.

[5] L. Alsedà, J. Llibre, and M. Misiurewicz, Combinatorial Dynamics and Entropy in Dimension One, vol. 5 of Advanced Series in Nonlinear Dynamics, World Scientific, River Edge, NJ, USA, 1993.

[6] M. Misiurewicz and Z. Nitecki, "Combinatorial patterns for maps of the interval," Memoirs of the American Mathematical Society, vol. 94, no. 456, pp. 1-112, 1991.

[7] L. Alsedà, J. Guaschi, J. Los, F. Mañosas, and P. Mumbrú, “Canonical representatives for patterns of tree maps," Topology, vol. 36, no. 5, pp. 1123-1153, 1997.

[8] X. Zhao, "On minimal fixed point numbers of relative maps," Topology and Its Applications, vol. 112, no. 1, pp. 41-70, 2001.

[9] H. Schirmer, "A survey of relative Nielsen fixed point theory," in Nielsen Theory and Dynamical Systems (South Hadley, Mass, USA 1992), vol. 152 of Contemporary Mathematics, pp. 291-309, American Mathematical Society, Providence, RI, USA, 1993.

[10] X. Zhao, "Estimation of the number of fixed points on the complement," Topology and Its Applications, vol. 37, no. 3, pp. 257-265, 1990.

[11] P. R. Heath and X. Zhao, "Nielsen numbers for based maps, and for noncompact spaces," Topology and Its Applications, vol. 79, no. 2, pp. 101-119, 1997.

[12] L. Block, "Periods of periodic points of maps of the circle which have a fixed point," Proceedings of the American Mathematical Society, vol. 82, no. 3, pp. 481-486, 1981. 